\title{
Alternate way to teach Fourier optics
}

\section{Henri Arsenault}

Henri H. Arsenault, "Alternate way to teach Fourier optics," Proc. SPIE 2525, 1995 International Conference on Education in Optics, (13 October 1995); doi: $10.1117 / 12.224070$

SPIE Event: SPIE's 1995 International Symposium on Optical Science, SPIE. Engineering, and Instrumentation, 1995, San Diego, CA, United States 


\title{
An alternate way to teach Fourier Optics
}

\author{
Henri H. Arsenault \\ COPL, département de physique, Université Laval,Québec, Qc., Canada GIK 7P4
}

\begin{abstract}
Scalar diffraction theory is usually introduced using the classical Green function approach as a solution to a boundary value problem. I propose a different approach first proposed by Duffieux and later put on a solid mathematical foundation by Arsac. and which exploits the close connection between Fourier theory and linear systems theory; it is based on elementary distribution theory, where scalar diffraction appears naturally as a convolution of the diffracting screen with an optical propagator. All the classical expressions such as Rayleigh's integral formula and the Kirchhoff-Sommerfeld diffraction integral are easily derived as special cases. The fact that diffraction appears naturally as a convolution facilitates the integration of diffraction theory with linear systems theory, which has come to play a major role in fourier optics.
\end{abstract}

\section{INTRODUCTION}

The classical way to teach Fourier optics is to begin with scalar diffraction theory based on the Green functions approach, then to consider different approximations until the Fresnel approximation is reached. A further approximation leads to Fraunhofer diffraction, where the diffraction pattern is the Fourier transform of the aperture. An excellent exposition of this theory is in the book by J. W.Goodman 1 . This book clearly points out the difficulties and the inconsistencies of this theory; these are not simple questions, as can be seen by the fact that in his classical book 2 , Jackson erroneously attributes the difficulties of the Green function approach to the fact that a scalar theory is being used, and wrongly claims that those difficulties disappear if a vector theory is used. The basic problem is that the Green function approach is well adapted to solving boundary value problems where the boundary values are known on a closed surface, which is not the case for the diffraction problem, where part of the boundary condition is the required solution.

In the old approach, some notions of linear systems theory (including Fourier theory) are often introduced, and Fresnel diffraction is shown to be expressible as a convolution of the diffracting aperture with a free space propagator of the form $\exp \left(\mathrm{ix}^{2} / \lambda \mathrm{d}\right)$. This links the theory of diffraction with the theory of linear systems, which is particularly useful for engineering students who are already familiar with the mathematical tools of linear systems theory including the basic theorems of Fourier theory. This approach was well described by J. Gaskill at the St Petersburg Conference on Education ${ }^{3}$. In his book based on his course oriented to engineers, Gaskill begins with linear systems theory, followed by fourier theory; diffraction theory and the theory of imaging are at the end.

In a recent conference in optics education meeting held in Pecs Hungary in 1993, four papers were presented on how to teach Fourier optics by non-conventional means, ranging from geometrical optics methods to operator algebra, which made it the most popular subject of the conference. The approach I present here was also presented at that conference, but because the number of participants was very small (I was the only participant from North America), and because the proceedings have not been widely distributed, I am presenting it again to make it available to a wider audience.

in this paper I propose a somewhat different approach that differs mostly in the manner in which diffraction theory is handled. Instead of the Green's function approach, I use an alternate approach using elementary distribution theory where the exact scalar solution to he Helmholtz equation appears as a convolution. Various approximations to this expression yield the various expressions of scalar diffraction theory such as Rayleigh's integral equations, the Kirchhoff formulae and the Fresnel and Fraunhofer approximations. The connection to linear systems theory is then natural and easy, because diffraction immediately appears as a linear filtering operation before any approximations are introduced. This approach avoids the erroneous impression of many students that the Fourier transform is related to diffraction theory only in the approximation of Fraunhofer diffraction. For many years I have been teaching a graduate course on Fourier optics that uses this formulation of diffraction.

That diffraction is a convolution appears immediately obvious when the conditions for a transformation to be 
expressible as a convolution are considered: a necessary and sufficient condition is that 1)the relationship between the input and the output satisfy the superposition principle, and 2) the output be invariant under a translation of the input. The first condition is satisfied by the fact that the wave equation is a linear differential equation, and the second is satisfied because of the isotropy of space.

It is paradoxical that the approach to diffraction theory based on this fact was not pursued until the 1960's, despite the fact that the founder of Fourier optics P. M. Duffieux proposed it in his book published in the 1940's and translated into English by me in 19834; unfortunately Duffieux did not have the mathematical tools (distribution theory) that were required, so his arguments relied heavily on qualitative and graphical arguments and he could not derive an expression for the propagator.

The exact convolution theory of scalar diffraction theory based on distribution theory was first proposed by Arsac in 19615. This French book was generally ignored, and has remained relatively unknown despite the publication of an English translation in the 70's. In the intervening years following Arsac's original work, a number of authors have published approximate 6,7 or exact 8,9 theories of diffraction using a convolution formulation. This approach to diffraction has been slightly noted with some interest, but so far no text on Fourier optics has integrated it in its presentation of diffraction. For example, in his book, Gaskill attributes it to Shack and Harvey ${ }^{10}$, who were apparently unaware of Arsac's book, and notes that it is much less restrictive than the alternate approach based on Green's functions.

A major advantage of this approach is that despite the use of elementary distribution theory, the mathematical derivations are shorter than those using the Green functions approach. The knowledge of distribution theory required is very limited, and nothing more complicated than 3D delta functions is required.

\section{DIFFRACTION AS A LINEAR FILTERING PROCESS}

We now introduce scalar diffraction theory as a linear filtering process. A more detailed and rigorous derivation is given in the book by Arsac, as well as modifications required to take into effect edge diffraction waves. in our proofs, we have emphasized simplicity at the expense of rigor.

\section{Forward diffraction}

Consider a monochromatic scalar wave field $f(x, y, z)$ in free space. The problem of diffraction is to determine the wave field in space, given the values of the field on the plane $\mathrm{z}=0$. The wave field in free space for quasi-monochromatic light satisfies the Helmholtz equation

$\frac{\partial^{2}}{\partial x^{2}} f(x, y, z)+\frac{\partial^{2}}{\partial y^{2}} f(x, y, z)+\frac{\partial^{2}}{\partial z^{2}} f(x, y, z)=\frac{k^{2}}{4 \pi^{2}}$

with $\mathrm{k}=2 \pi / \lambda$. the Fourier transform of this expression is

$\left(u^{2}+v^{2}+w^{2}-\frac{k^{2}}{4 \pi^{2}}\right) F(u, v, w)=0$

where $(\mathrm{u}, \mathrm{v}, \mathrm{w})$ are the conjugate coordinates corresponding to the spatial coordinates $(\mathrm{x}, \mathrm{y}, \mathrm{z})$, and $\mathrm{F}(\mathrm{u}, \mathrm{v}, \mathrm{w})$ is the Fourier transform of $f(x, y, z)$.

Arsac has pointed out that Eq. (2) implies that in Fourier space, $F(u, v, w)$ is zero everywhere except on the surface of a sphere of radius $\mathrm{k} / 2 \pi$. Therefore this may be expressed

$\mathrm{F}\left(\mathrm{u}, \mathrm{v}, \mathrm{w}_{0}\right) \delta\left[\mathrm{w}-\left(\frac{\mathrm{k}^{2}}{4 \pi^{2}}-\mathrm{u}^{2}-\mathrm{v}^{2}\right)^{\frac{1}{2}}\right]=\mathrm{F}(\mathrm{u}, \mathrm{v}, \mathrm{w})$.

Now the wave field $f(x, y, z)$ may be written as the fourier transform

$f(x, y, z)=\iiint F(u, v, w) e^{-2 \pi i(u x+v y+w z)} d u d v d w$. 
inserting Eq. (3) into this expression allows the reduction of the integral to a double integral

$f(x, y, z)=\iint F_{0}(u, v) e^{-2 \pi i w_{0} z} e^{-2 \pi i(u x+v y)} d u d v$

where $\mathrm{w}_{0}=\left(\mathrm{k}^{2} / 4 \pi^{2}-\mathrm{u}^{2}-\mathrm{v}^{2}\right)$, and $\mathrm{F}_{0}(\mathrm{u}, \mathrm{v})=\mathrm{F}\left(\mathrm{u}, \mathrm{v}, \mathrm{w}_{0}\right)$.

Setting $\mathrm{z}=0$ in Eq. (5) yields

$f(x, y, z)=\iint F_{0}(u, v) e^{-2 \pi i(u x+v y)} d u d v$.

So $F_{0}(u, v)$ is the Fourier transform of the field $f(x, y, 0)$ on the diffracting plane at $z=0$. From this fact and from the convolution theorem, Eq. (5) may be expressed as the convolution

$\mathrm{f}(\mathrm{x}, \mathrm{y}, \mathrm{z})=\mathrm{f}(\mathrm{x}, \mathrm{y}, 0) * \mathrm{~g}(\mathrm{x}, \mathrm{y}, \mathrm{z})$,

where $\mathrm{g}(\mathrm{x}, \mathrm{y}, \mathrm{z})$ is the Fourier transform of $\exp \left(-2 \pi \mathrm{iw} \mathrm{w}_{0} \mathrm{z}\right)$. This Fourier transform has been evaluated before 11 and is equal to

$g(x, y, z)=\frac{1}{2 \pi} \frac{\partial}{\partial z}\left(\frac{e^{i k \rho}}{\rho}\right)$

where $\rho=\left(x^{2}+y^{2}+z^{2}\right)^{1 / 2}$.

Equation (9) expresses the diffracted amplitude as a convolution of the field $f(x, y, 0)$ on the diffracting plane with a function that is the diffracted field of a point source, as may be easily verified by setting

$f(x, y, 0)=\delta(x, y)$

in Eq. (8). This impulse response is the wave propagator for scalar diffraction. We can use the values of the field $\mathrm{g}(\mathrm{x}, \mathrm{y}, \mathrm{z})$ given by Eq. (9) and set $\mathrm{R}=\left[\left(\mathrm{x}-\mathrm{x}_{0}\right)^{2}+\left(\mathrm{y}-\mathrm{y}_{0}\right)^{2}+\mathrm{z}^{2}\right]^{1 / 2}$ to get the expression

$f(x, y, z)=\int_{\Pi} \int F\left(x_{0}, y_{0}, 0\right) \frac{\partial}{\partial z}\left[\frac{e^{i k R}}{R}\right] d x_{0} d y_{0}$,

which is one of Rayleigh's well-known integral formulae for diffraction.

\section{Diffraction between two arbitrary planes}

The above theory can be easily modified to obtain an expression for diffraction between two arbitrary planes $\mathrm{z}=\mathrm{z}_{0}$ and $\mathrm{z}=\mathrm{z}_{1}$ : instead of setting $\mathrm{z}=0$ on the diffraction plane, set it equal to $\mathrm{z}_{0}$; the convolution expression then becomes

$f\left(x, y, z_{1}\right)=f\left(x, y, z_{0}\right) * \frac{\partial}{\partial z_{1}}\left[\frac{e^{i k \rho_{0}}}{\rho_{0}}\right]$

where $\rho_{0}=\left[x^{2}+y^{2}+\left(z_{1}-z_{0}\right)^{2}\right]^{1 / 2}$, which expresses the diffracted field in a plane $z=z_{1}$ as a function of the field at the plane $\mathrm{z}=\mathrm{z}_{0}$.

\section{Inverse diffraction}

In the previous derivation, we made no suppositions regarding whether $z_{1}>z_{0}$. Therefore Eq. (12) is valid whether $z_{1}>z_{0}$ or not. Therefore when $z_{1}<z_{0}$, Eq. (12) is the solution to the inverse problem of diffraction, namely to find what field is required on plane $z_{0}$ to obtain a given field on plane $z_{1}$. If the condition

$\mathrm{k}^{2} / 4 \pi^{2}>\mathrm{u}^{2}+\mathrm{v}^{2}$

is satisfied, there is no problem, because only homogeneous waves are present. But if the condition is not satisfied, the waves are non-homogeneous. For forward diffraction, the corresponding waves are 
evanescent waves propagating in a direction parallel to the plane $\mathrm{z}=\mathrm{z}_{0}$, whose amplitudes decrease exponentially in the direction of increasing $\mathrm{z}$. But in the case of inverse diffraction, these waves become exponentially divergent waves that cannot be generated in a passive medium.

\section{HOW TO TEACH IT}

Although there is more than one way to teach Fourier optics using this method, depending on previous knowledge of the students, here is how I do it in a beginning graduate course. I assume no previous knowledge of Fourier theory on the part of the students.

Because the mathematical derivations use Dirac delta functions and basic Fourier theorems, I begin the course with an introduction to basic Fourier theory. in the first hour, I establish the connection between the Fourier transform and linear systems by showing that the complex exponential exp(iux) is an eigenfunction of a linear shift invariant system. The basic theorems and elementary generalized functions or distributions such as the delta function are then introduced. No additional knowledge of distribution theory is required. The material contained int he early chapters of the book by Bracewell 12 are quite sufficient. This is followed by diffraction theory as summarized above, and continues with topics such as discrete Fourier transforms, the linear theory of image formation, spatial frequency filtering, coherent and incoherent transfer functions, applications of Fourier theory to stochastic processes and other examples. Optical information processing and optical computing are covered in a separate course.

For students with a good background in Fourier theory and in linear systems, such as most students with an electrical engineering background, the course could begin directly with the diffraction theory described above, after a short review of linear systems.

\section{CONCLUSION}

The formulation of scalar diffraction theory using distribution theory, in addition to avoiding the pitfalls associated with classical scalar diffraction theory, yields results that express diffraction as a linear filtering process and easily integrates diffraction theory into the context of linear systems theory and Fourier theory.

\section{REFERENCES}

J. W. Goodman, An introduction to Fourier optics, McGraw-Hill , NY (1968).

2.D.Jackson, Classical Electrodynamics, John Wiley and Sons, N.Y. (1963).

3. J. Gaskill, "The role of Fourier optics in optics education", in Education in Optics, G. B. Altshuler and B. J. Thompson, Editors, proc. SPIE 1603, pp. 78-84 (1992).

4.P. M. Duffieux, The Fourier transform and its applications to optics, John Wiley and Sons, NY (1983).

5. J. Arsac, Transformation de Fourier et théorie des distributions, Dunod, Paris (1961).

6. J. T. Winthrop and C. R. worthington, J. Opt. Soc. Am. 56, 588 (1966).

7.J. T. Winthrop and C. R. Worthington, J. Opt. Soc. Am. 56, 1362 (1966).

8.G. C. Sherman, J. Opt. Soc. Am. 57, 1490 (1967). 
9. J. R. Shewell and E. Wolf, J. Opt. Soc. Am. 58, 1596 (1968).

10. V. Shack and V. E. Harvey, "An investigation into the distribution of radiation scattered by optical surfaces”, Optical Sciences Center (University of Arizona), final report (1975).

11. J. Banos Jr., Radiation in the presence of a conducting half-space, Pergamon Press, Oxford (1966).

12. R. N. Bracewell, The fourier transform and its applications, McGraw-Hill, NY (1978). 